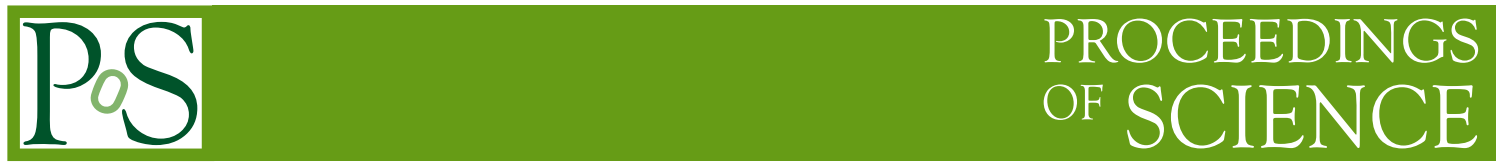

\title{
Lower orphan afterglow rates for Gaia and LSST
}

\author{
József Kóbori* \\ Eötvös University, Budapest, Hungary \\ E-mail: jkobori@elte.hu
}

\section{Zsolt Bagoly}

Eötvös University, Budapest, Hungary

\section{Lajos G. Balázs}

Eötvös University, Budapest, Hungary

TA CSFK Konkoly Observatory, Budapest, Hungary

\section{Željko Ivezić}

University of Washington, Seattle, USA

\section{Istvan Horvath}

National University of Public Service, Budapest, Hungary

\begin{abstract}
Gamma-ray bursts are high energy transients accompanied by their afterglows produced in relativistic shocks. The outflow is often highly collimated into a narrow jet. If the jet's symmetry axis is not pointed towards the observer due to relativistic effects the observer can not detect radiation from this beaming cone, but later on, in the afterglow phase the relativistic beaming of the jet becomes less significant, and the jet might be visible in the optical/radio bands. These bursts (the promp $\gamma$-emission is not detected, only the afterglow) are the so-called orphan GRBs. In this work we predict the rate of orphan-to-not-orphan bursts for the Gaia and LSST sky survey programs: our results suggest lower rates by $\sim 3$ times for LSST and $\sim 9$ times for Gaia.
\end{abstract}

Swift: 10 Years of Discovery

2-5 December 2014

La Sapienza University, Rome, Italy

\footnotetext{
${ }^{*}$ Speaker.
} 


\section{Introduction}

There are theoretical considerations that gamma-ray bursts (GRBs) produce highly relativistic outflows into a narrow jet ([1]). When the ejected material encounters the circumburst medium of the progenitor synchrotron radiation is produced observed as an afterglow. The case when the burst is viewed off-axis and only the afterglow is detected is called an orphan-afterglow observation $([2,3])$. Detection of such bursts would constrain the ratio of orphan to not-orphan bursts. In this article we make a prediction for the orphan afterglow rate using the observational strategy of the Large Synoptic Sky Survey ([7]) and the Gaia satellite.

\section{Simulations}

The simulation of GRB afterglows was carried out with the numerical code BOXFIT developed by Hendrik van Eerten ([4]). The input parameters of the afterglows were chosen according to their observed or theoretical distributions. The initial jet half opening angle $\left(\theta_{0}\right)$ was chosen from the range $\sim 3^{\circ}-\sim 28^{\circ}$ and we applied two different types of distributions: a uniform $\left(P\left(\theta_{0}\right) \propto \cos \left(\theta_{0}\right)\right)$ and a power-law $\left(P\left(\theta_{0}\right) \propto \theta_{0}^{-1}\right)$ distribution. The redshift varies between the values of 0 and 10, the type of the distribution is taken from Wanderman et al. 2010 ([5]).

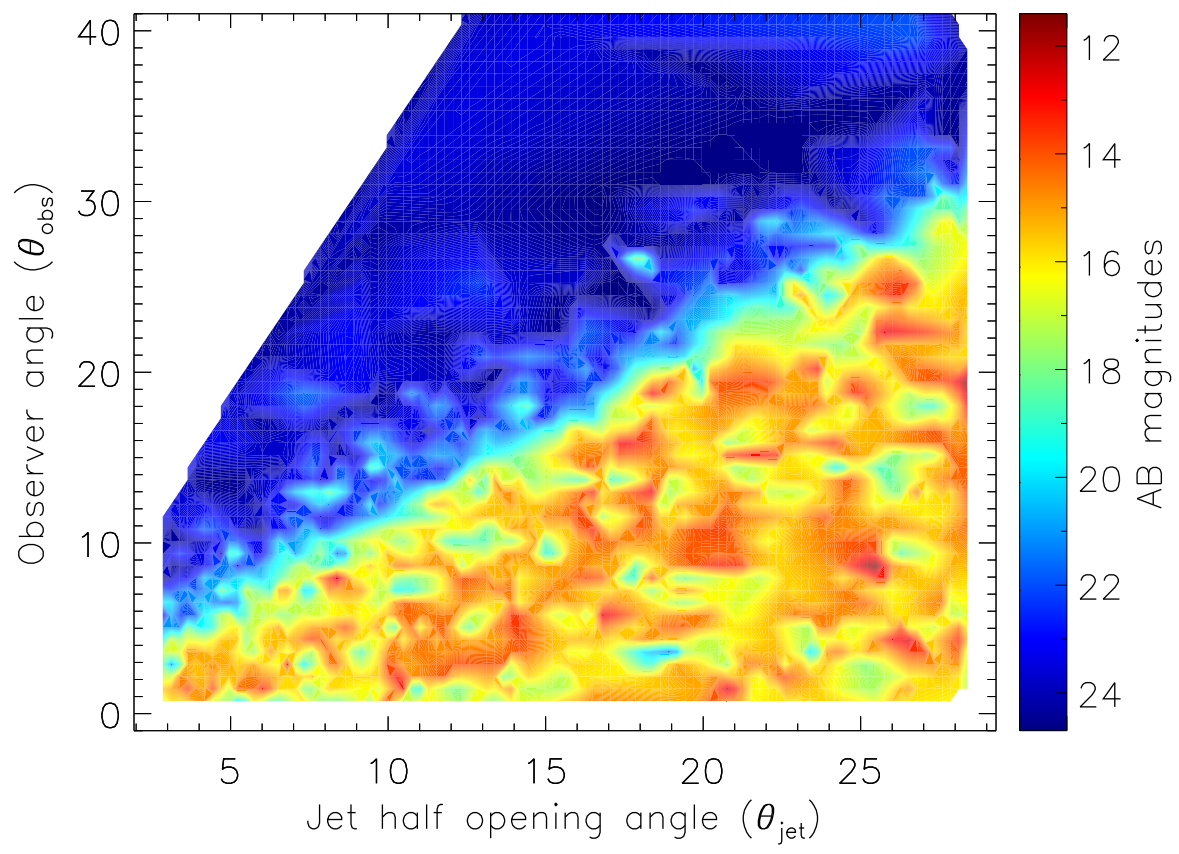

Figure 1: This contour plot shows how the peak magnitude of the afterglows correlate with $\theta_{0}$ and $\theta_{\mathrm{obs}}$ when $P\left(\theta_{0}\right) \propto \theta^{-1}$. As it can be seen the peak magnitude sharply drops when $\theta_{\text {obs }}$ becomes larger than $\theta_{0}$. This is the consequence of the fact that the energy remains enclosed inside the initial opening angle of the jet. Taken $P\left(\theta_{0}\right) \propto \cos (\theta)$ the result is almost identical. 


\section{Intrinsic rate of GRBs}

We assume that the intrinsic rate of GRBs follows the one determined by Nakar et al. (2002) ([6]). According to their results, the BATSE detection rate of long bursts with $\theta_{\text {obs }}<\theta_{0}$ is 667 GRB/year. In order to determine the expected ratio of orphan to not-orphan GRBs $\left(N_{\text {on }}\right)$, we generated $\theta_{\mathrm{obs}}$ and $\theta_{0}$ pairs and then counted the cases where the observer angle is smaller (or larger) than the initial jet half opening angle $\left(\theta_{\mathrm{obs}}<\theta_{0}\right.$ or $\left.\theta_{\mathrm{obs}}>\theta_{0}\right)$ :

$$
\begin{aligned}
& \text { - } N_{\text {on }} \approx 5.33 \text {, if } P\left(\theta_{0}\right) \propto \theta_{0}^{-1}, \\
& \text { - } N_{\text {on }} \approx 3.88 \text {, if } P\left(\theta_{0}\right) \propto \cos \left(\theta_{0}\right) .
\end{aligned}
$$

\section{General results}

First, regardless of any sky survey program, we determined how the afterglows' peak brightness depends on $\theta_{0}$ and $\theta_{\mathrm{obs}}$, it is shown on Fig. 1: the farther the observer from the jet symmetry axis, the lower the brightness of the afterglow.

\section{Orphan afterglows - LSST}

The telescope will cover the observable sky from it's site $\left(\sim 10000 \mathrm{deg}^{2}\right)$ every three days ([7]), thus, the probability that a light curve will be observed by the LSST equals 1 if an afterglow will be brighter for at least 3 days than the LSST's detection limit in any band. Based on simulations the bandpass $r$ is the most suitable for observing GRB afterglows in case of the LSST .

Considering afterglows both with uniform $\theta_{0}$ and power-law $\theta_{0}$ distribution case from the redshift range $z \in[0,10]$ and applying all of the observational constraints the LSST will detect $\sim 2$ optical afterglows every three days (one orphan and one not-orphan). Taking into account the flux attenuation by the Ly- $\alpha$ absorption line shifting into the $r$ band, i.e. making the cut in the sample at redshift $z \simeq 3.5$, the number of observable afterglows per three days reduces to $\simeq 1$ ( $\simeq 0.5$ orphan and $\simeq 0.5$ not-orphan).

In Fig. 2 we show the histogram of the $t_{\mathrm{obs}}$ values (the time duration while an afterglow is brighter than the LSST'S detection limit) for the case when $\theta_{0}$ has a uniform distribution $\left(P\left(\theta_{0}\right) \propto\right.$ $\cos \left(\theta_{0}\right)$ ). When plotting the afterglows' peak brightness against the redshift (Fig. 3) we can see that GRBs with higher redshift still could be detected because of their high brightness. The reason we can not detect such GRBs is that the interviening neutral hydrogen gas clouds attenuate the afterglows' flux, reducing the probability to observe them.

\section{Orphan afterglows - Gaia}

There are two requirements for an afterglow to be observed by Gaia:

1. its peak brightness has to reach 20 magnitudes, which is the detection limit of the telescope,

2. the celestial coordinate of a GRB has to lie on the scanning track of the telescope. 


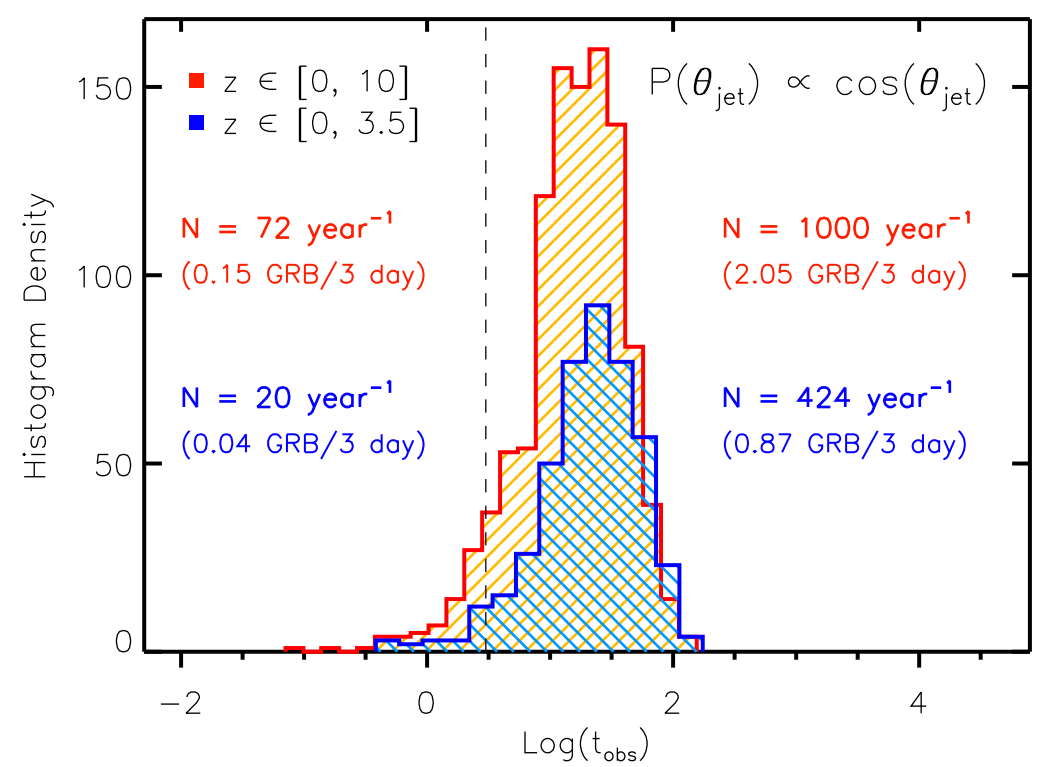

$[\mathrm{h} !]$

Figure 2: The histogram shows the distribution of time intervals $\left(t_{\mathrm{obs}}\right)$ while the afterglows have a flux level above the LSST's detetction limit, $\theta_{0}$ has a uniform distribution in this case (with power-law distribution the numbers are as follows: $1034+97$ and $444+27$ bursts/year if $z \in[0,10]$ and $z \in[0,3.5]$, respectively.) If $t_{\mathrm{obs}} \geq 3$, then the afterglows will be observed probability of 1 . The colors represent the afterglow subsamples: the red histogram shows GRBs from the redshift range $[0,10]$, while the blue one corresponds to a more realistic model, where we take into account the effect of the Lyman- $\alpha$ absorption in the spectrum, i.e. considering afterglows only up to $\simeq 3.5$ (corresponds to the LSST's $r$-band). The vertical dashed line separates the afterglows where $t_{\mathrm{obs}}<3$ days and $t_{\mathrm{obs}} \geq 3$ days.

Considering only GRBs with power-law $\theta_{0}$ distribution (4222 burst/year) 788 are bright enough to be detected by Gaia. Applying the scanning law only seven bursts is detectable, but the flux suppression of Ly- $\alpha$ systems towards the GRBs reduces this number further to one burst/year, which is not an orphan burst. This rate is significantly lower than those predicted by Rossi et al. 2008 ([8]): $\approx 8.8 \mathrm{GRB} /$ year. As for the LSST we also plotted the $\theta_{0}-\theta_{\text {obs }}-$ peak magnitude variables on the contour plot for the Gaia system on Fig 4. 


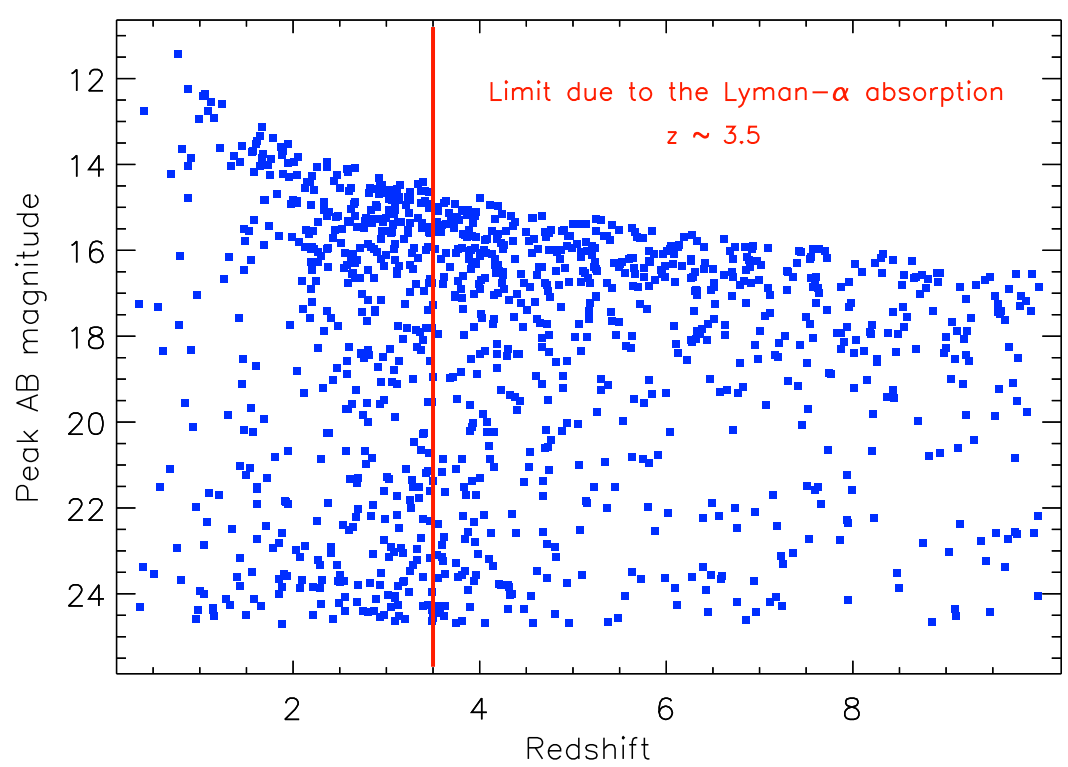

Figure 3: The figure shows the distribution of $\mathrm{AB}$ peak magnitudes in the $r$ band against the redshift for light curves when $P\left(\theta_{0}\right) \propto \theta_{0}^{-1}$ for the LSST. As the redshift becomes higher, the peak brightness becomes lower. The reason we do not observe GRBs from high redshifts with 15-16 peak magnitudes is the flux suppression by the interviening neutral hydrogen gas clouds. The vertical red line indicates the redshift limit where the LSST's $r$-band filter overlaps the $1217 \AA$ Ly- $\alpha$ absorption line, therefore, the afterglows start to fade because of the Ly- $\alpha$ forest suppression. The higher density of GRBs at $z \simeq 3.5$ is the result of the applied redshift distribution.

\section{Conclusion}

From the results above we can draw the following conclusions:

1. taking into account the Ly- $\alpha$ absorption by the interviening hydrogen systems the number of detectable afterglows reduces by a factor of two in the case of LSST, and this number changes from seven to one when observing with Gaia

2. the distribution type of the initial jet opening angle $\left(\theta_{0}\right)$ is almost irrelevant,

3. similarly, calculations with uniform and log-uniform burst energy distributions give comparable results,

4. LSST will detect one orphan and one not - orphan afterglows every three nights,

5. Gaia will detect one afterglows every year.

Acknowledgements: This work was supported by the Hungarian OTKA-77795 and OTKA111016 grants. Development of the Boxfit code was supported in part by NASA through grant NNX10AF62G issued through the Astrophysics Theory Program and by the NSF through grant AST-1009863. 


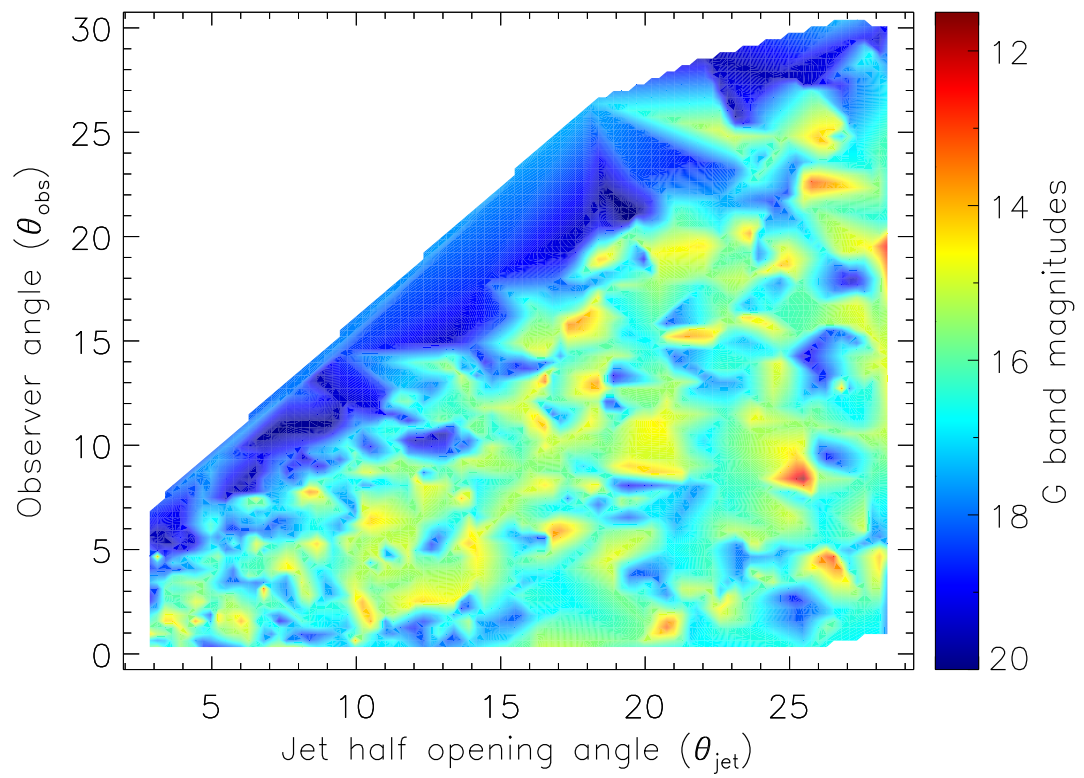

Figure 4: The contour plot shows how the maximal brightness of afteglows detectable by Gaia depends on the observer's angle $\left(\theta_{\mathrm{obs}}\right)$ and initial jet half opening angle $\left(\theta_{0}\right)$. It is clearly seen that if the observer is inside the cone of the jet the peak magnitude can be higher. As soon as $\theta_{\mathrm{obs}}>\theta_{0}$ the peak brightness sharply drops, and the afterglow becomes undetectable.

\section{References}

[1] J. E. Rhoads, How to tell a jet from a balloon: a proposed test for beaming in gamma-ray bursts, ApJ, 487, [arXiv:astro-ph/9705163]

[2] J. E. Rhoads, The dynamics and light curves of beamed gamma-ray burst afterglows, ApJ, 525, [arXiv:astro-ph/?]

[3] J. Granot, A. Panaitescu, P. Kumar, S. E. Woosleys, Off-axis afterglow emission from jetted gamma-ray bursts, ApJL, 570, [arXiv:astro-ph/?]

[4] H. van Eerten, A. van der Horst, A. MacFadyen, Gamma-ray burst afterglow broadband fitting based directly on hydrodynamics simulations, ApJ, 749, [arXiv: astro-ph/?]

[5] D. Wanderman, T. Piran, The luminosity function and the rate of Swift's gamma-ray bursts, MNRAS, 406, [arXiv:astro-ph/?]

[6] E. Nakar, T. Piran, J. Granot, The detectability of orphan afterglows, ApJ, 579, [arXiv:astro-ph/?]

[7] LSST Science Collaboration, LSST Science Book, Version 2.0, [arXiv: astro-ph/?]

[8] E. M. Rossi, R. Perna, F. Daigne, 'Orphan' afterglows in the Universal structured jet model for gamma-ray bursts, MNRAS, 390, [arXiv:astro-ph/?] 